\title{
ON AN ORDINARY DIFFERENTIAL EQUATION INVOLVING A CONVEX FUNCTION( $\left.{ }^{1}\right)$
}

\author{
BY \\ PHILIP HARTMAN
}

1. Introduction. The differential equation to be considered below is

$$
r^{\prime}+G(t, r)+q(t)=0,
$$

where $r^{\prime}=d r / d t, G(t, r)$ and $q(t)$ are continuous functions for $-\infty<r<\infty$ and $0 \leqq t<\omega(\leqq \infty)$. In the main results, it will be assumed that either $G(t, r)$ is a convex function of $r$ (for $t$ fixed) or that $G(t, r)$ has a suitable convex minorant $g(r)$, independent of $t$.

When $G(t, r)=r^{2},(1.1)$ becomes the Riccati equation

$$
r^{\prime}+r^{2}+q(t)=0 \text {. }
$$

In this case, $r=r(t)$ is a solution of (1.2) if and only if $r$ is the logarithmic derivative of a solution $x=x(t)$ of the second order linear equation

$$
x^{n}+q(t) x=0 .
$$

Thus, the existence of a solution of (1.2) for $t$ near $\omega$ is equivalent to the nonoscillatory character of (1.3) at $t=\omega$. The case $G(t, r)=|r|^{a}$ of (1.1),

$$
r^{\prime}+|r|^{a}+q(t)=0, \text { where } a>1,
$$

occurs in a number of places; cf., e.g., [5, pp. 226-227], where $\omega=\infty, q(t) \geqq 0$, and the question of the existence of a solution $r(t)>0$ of (1.4) for large $t$ is discussed in connection with the study of a binary system of differential equations near a stationary point of a certain type. It was pointed out there that a number of results known for (1.2) have analogues for (1.4). Cf. also [6].

In $\S 2, \omega=\infty$ and we collect some basic facts concerning conditions necessary or sufficient for (1.1) to have solutions $r=r(t)$ for large $t$, and for such solutions to satisfy $r(t) \rightarrow 0$ as $t \rightarrow \infty$ or $\int^{\infty} G(t, r(t)) d t<\infty$. The proofs are adapted from the Riccati case (1.2) and are only indicated.

In $\S 3, \omega=\infty$ and we derive some further necessary conditions for the existence of solutions of (1.1) for large $t$. Although, Theorem 3.1 is known for the case (1.2), the proof in this particular case (1.2) depends on the relationship between (1.2)

Received by the editors April 22, 1969.

( $\left.{ }^{1}\right)$ Research partially supported by the Air Force Office of Scientific Research, Office of Aerospace Research, United States Air Force, under AFOSR Contract No. F44620-67-C-0098. 
and (1.3), and cannot be carried over directly to (1.1). Theorem 3.2 seems to be new even for (1.2) and implies, in this case, that if $q(t) \geqq 0$, then a necessary condition for (1.3) to be nonoscillatory at $t=\infty$ is that

$$
\int^{\infty} t \exp \left(-4 \int^{t} q^{1 / 2}(s) d s\right) d t=\infty
$$

(The example $q(t)=1 / 4 t^{2}$ shows that 4 cannot be replaced by a larger constant.)

The main novel results of the paper are in $\$ 4$. Here we introduce the concept of a principal solution $r=r_{*}(t)$ for $(1.1)$ at $t=\omega$ under suitable conditions on $G$. (This generalizes and gives a new simple proof for the existence of principal solutions for equations (1.3), nonoscillatory at $t=\omega$.) Standard results on the cross-ratios of solutions of (1.2) suggest the consideration of $\left[r^{2}(t)-r^{0}(t)\right] /\left[r^{1}(t)-r^{0}(t)\right]$, where $r^{0}<r^{1}<r^{2}$ are solutions of (1.1) for $t$ near $\omega$. It is noted that this ratio has a limit $L$, as $t \rightarrow \omega$. Most of this section deals with two related questions: one, the derivation of conditions on $G, q$ which assure that $L=1$ if $r^{0}=r_{*}(t)$ is the principal solution and $r^{1}, r^{2}$ are arbitrary [as in the case for (1.2)] and two, the comparison of solutions of (1.1) and $r^{\prime}+G(t, r)+q_{0}(t)=0$, when $\left|q-q_{0}\right|$ is "small". A byproduct of the theorems obtained is a result for (1.3): if (1.3) is nonoscillatory at $t=\omega$ and $x=x^{0}(t), x^{1}(t)$ are principal, nonprincipal solutions at $t=\omega$, then

$$
\int^{\omega} d t /\left|x^{0}(t) x^{1}(t)\right|=\infty
$$

(A direct proof of this is quite simple, but it does not seem to have been stated before.)

It will always be assumed that $G(t, r), q(t), q_{0}(t), p(t)>0$ are continuous for $0 \leqq t<\omega(\leqq \infty)$ and arbitrary $r$. While this assumption of continuity will not be repeated below, other conditions will be stated as needed.

2. Preliminary results. In this section $\omega=\infty$, so that $t$ varies over the half-line $[0, \infty)$. The analogue of the Sturm comparison theorem is the following:

Proposition 2.1. Assume that $G(t, r) \geqq 0$. Then the differential equation (1.1) has a solution for large $t$ if and only if there exists a function $v=v(t)$ of class $C^{1}$ for large $t$ satisfying

$$
v^{\prime}+G(t, v)+q(t) \leqq 0
$$

(in which case, the maximal solution of (1.1) satisfying an initial condition $r\left(t_{0}\right) \geqq v\left(t_{0}\right)$ exists for $\left.t \geqq t_{0}\right)$. In particular, if

$$
r^{\prime}+G(t, r)+q_{0}(t)=0
$$

has a solution and

$$
q(t) \leqq q_{0}(t)
$$

large $t$, then (1.1) has a solution for large $t$. 
The proof follows from standard theorems on differential inequalities and the fact that if a solution $r=r(t)$ of (1.1) exists on $S<t<T(<\infty)$, but not on $S<t \leqq T$, then $r(t) \rightarrow-\infty$ as $t \rightarrow T-0$.

Proposition 2.2. Let $m=m(r)$ be a continuous, nondecreasing function for $r \geqq 0$ satisfying

$$
m(r)>0 \text { for } r>0 \text { and } \int^{\infty} d r / m(r)<\infty
$$

and let

$$
G(t, r) \geqq 0 \text { for all } r, \quad G(t,-r) \geqq m(r) \text { for large } r>0 .
$$

If $q(t) \geqq 0$ and (1.1) has a solution $r=r(t)$ for large $t$, then $r(t) \geqq 0$ and

$$
Q(t)=\int_{t}^{\infty} q(s) d s=\lim _{T \rightarrow \infty} \int_{t}^{T} q(s) d s \text { exists. }
$$

Proposition 2.3. Let $G$ and $m$ be as in Proposition 2.2. Assume that (2.6) holds (although $q(t) \geqq 0$ need not) and that (1.1) has a solution $r=r(t)$ for large $t$. Then

$$
\begin{gathered}
\int^{\infty} G(t, r(t)) d t<\infty, \\
r(t)=\int_{t}^{\infty} G(s, r(s)) d s+Q(t) \rightarrow 0, \text { as } t \rightarrow \infty .
\end{gathered}
$$

The last two assertions are due to Wintner [10] in the Riccati case $G(t, r)=r^{2}$ and his indicated proof can be adapted here.

Proposition 2.4. Let $G$ and $m$ be as in Proposition 2.2 and, in addition,

$$
\begin{array}{ll}
G(t, r) \rightarrow 0 & \text { as }(t, r) \rightarrow(\infty, 0), \\
G(t, r) \geqq m(|r|) & \text { for all } r .
\end{array}
$$

Let (1.1) have solutions for large $t$. Then a necessary and sufficient condition that one (or every) solution of (1.1) for large $t$ satisfy $r(t) \rightarrow 0$ as $t \rightarrow \infty$ is that

$$
\sup _{0<v<\infty}(1+v)^{-1}\left|\int_{t}^{t+v} q(s) d s\right| \rightarrow 0 \text { as } t \rightarrow \infty \text {. }
$$

See, e.g., the proof of Lemma 1 in [4, pp. 392-394] (which is adapted from [2]). A partial converse for Proposition 2.3 is the following:

Proposition 2.5. Assume that $G \geqq 0$ satisfies (2.9) and

$$
G(t, r) \text { is a convex function of } r \text { (for fixed } t) \text {. }
$$


Let (1.1) have a solution $r=r(t)$ for large $t$. Then a necessary condition for (2.7) is that there exist a constant $C$ such that

$$
Q(t)=C-\int_{0}^{t} q(s) d s
$$

satisfies, for $\lambda>1$,

$$
T^{-1} \int_{0}^{T} G(t, Q(t) / \lambda) d t \rightarrow 0 \text { as } T \rightarrow \infty .
$$

If (2.12) is replaced by the condition

$$
G(t, r) \geqq g(r), \text { where } g(r) \text { is convex, }
$$

then (2.14) is replaced by

$$
T^{-1} \int_{0}^{T} g(Q(t) / \lambda) d t \rightarrow 0 \text { as } T \rightarrow \infty,
$$

and if $g(r)>0$ for $r \neq 0$, then

$$
\lim _{t \rightarrow \infty} T^{-1} \int_{0}^{T} d t\left(\int_{0}^{t} q(s) d s\right)=C \text { exists. }
$$

For the case $G(t, r)=r^{2}$, see [4] (or [5, p. 365]).

Proof. Let $r=r(t)$ be a solution of (1.1) for $t \geqq S$ satisfying (2.7). Then a quadrature of $(1.1)$ over $[S, t]$ gives

$$
r(t)+\int_{S}^{t} G(s, r(s)) d s+\int_{S}^{t} q(s) d s=r(S)
$$

which can be written as

$$
r(t)-\int_{t}^{\infty} G(s, r(s)) d s=Q(t)
$$

where $Q$ is given by (2.14) and

$$
C=r(S)-\int_{S}^{\infty} G(s, r(s)) d s+\int_{0}^{s} q(s) d s .
$$

By (2.19) and (2.12),

$$
0 \leqq G(t, Q(t) / \lambda) \leqq\left[G(t, r)+(\lambda-1) G\left(t,-\int_{t}^{\infty} G(s, r(s)) d s /(\lambda-1)\right)\right] / \lambda .
$$

It is clear that (2.14) follows from (2.7) and (2.9).

For a convex function $g,(2.19)$ implies that

$$
g(Q(t) / \lambda) \leqq\left[g(r)+(\lambda-1) g\left(-\int_{t}^{\infty} G(s, r(s)) d s /(\lambda-1)\right)\right] / \lambda
$$

and if (2.15) holds,

$$
0 \leqq g(t, Q(t) / \lambda) \leqq\left[G(t, r)+(\lambda-1) G\left(t,-\int_{t}^{\infty} G(s, r(s)) d s /(\lambda-1)\right)\right] / \lambda .
$$


Thus (2.16) follows from (2.7) and (2.9). By Jensen's inequality,

$$
0 \leqq g\left(T^{-1} \int_{0}^{T} Q(t) d t / \lambda\right) \leqq T^{-1} \int_{0}^{T} g(Q / \lambda) d t,
$$

and the right side tends to 0 as $T \rightarrow \infty$, by (2.15) and (2.16). From the condition $g(r)>0$ for $r \neq 0$,

$$
T^{-1} \int_{0}^{T} Q(t) d t \rightarrow 0 \text { as } T \rightarrow \infty
$$

This gives (2.17).

Proposition 2.6. Let $G \geqq 0$ satisfy (2.15), where

$$
g(r) \text { is convex, } g(-r)=g(r),
$$

and there exist continuous positive functions $g_{0}(s), g_{1}(r)$ for small $s>0$ and large $r>0$, respectively, satisfying

$$
g(r s) \geqq g_{0}(s) g_{1}(r), \quad \int^{\infty} \operatorname{tg}_{0}(1 / t) d t=\infty, \quad \int^{\infty} d r / g_{1}(r)<\infty .
$$

Assume that (1.1) has a solution $r=r(t)$ for large $t$. Then a sufficient condition for (2.7) is

$$
\liminf _{T \rightarrow \infty} T^{-1} \int_{0}^{T} d t\left(\int_{0}^{t} q(s) d s\right)>-\infty .
$$

For the case $G(t, r)=r^{2}$, see [4] (or [5, p. 365]). This proposition is applicable to $g(r)=|r|^{a}$ with $g_{0}(s)=s^{a}, g_{1}(r)=r^{a}$, provided that $1<a \leqq 2$. The proof of Proposition 2.6 is similar to the case $G(t, r)=r^{2}$ in [4] (cf. [5, pp. 365-366]), and the generalizations corresponding to [1] can be carried over to the present situation.

Proof. By (2.18) and (2.22),

$$
(t-S)^{-1} \int_{S}^{t} r(s) d s+(t-S)^{-1} \int_{S}^{t} d s\left(\int_{S}^{s} G(u, r(u)) d u\right) \leqq \text { const. }
$$

Suppose that (2.7) fails to hold. Then, for large $t, G \geqq 0$ implies that

$$
-(t-S)^{-1} \int_{S}^{t} r(s) d s \geqq \frac{1}{2}(t-S)^{-1} \int_{S}^{t} d s\left(\int_{S}^{s} G(u, r(u)) d u\right) \geqq 0 .
$$

By the convexity of $g$,

$$
(t-S)^{-1} \int_{S}^{t} g(r(s)) d s \geqq g\left[(t-S)^{-1} \int_{S}^{t} r(s) d s\right] .
$$

The last two inequalities and $g(r)=g(-r)$ give

$$
(t-S)^{-1} \int_{S}^{t} g(r(s)) d s \geqq g\left[\frac{1}{2}(t-S)^{-1} \int_{S}^{t} d s\left(\int_{S}^{s} G(u, r(u)) d u\right)\right] .
$$


Thus, if we put

$$
U(t)=\int_{S}^{t} d s\left(\int_{S}^{s} G(u, r(u)) d u\right)
$$

we have $U(t) \rightarrow \infty$ as $t \rightarrow \infty$ and, for large $t, U^{\prime} \geqq \int_{S}^{t} g(r(s)) d s$,

$$
(t-S)^{-1} U^{\prime} \geqq g[U / 2(t-S)] \geqq g_{0}(1 / 2(t-S)) g_{1}(U),
$$

or equivalently, $U^{\prime} / g_{1}(U) \geqq(t-S) g_{0}(1 / 2(t-S))$. In view of $(2.21)$, this leads to a contradiction and proves Proposition 2.6.

If the conditions (2.9), (2.15), (2.20) and (2.21) on $G(t, r)$ and $g(r)$ in both Propositions 2.5 and 2.6 hold and if (1.1) has solutions for large $t$, then it follows that (2.22) is equivalent to the existence of a constant $C$ such that (2.13) satisfies (2.16)-(2.17). Thus, we have

COROLlaRY 2.1. Let $G, g$ satisfy (2.9), (2.15), (2.20) and (2.21). Then a necessary condition for (1.1) to have a solution for large $t$ is either that

$$
\liminf _{T \rightarrow \infty} T^{-1} \int_{0}^{T} d t\left(\int_{0}^{t} q(s) d s\right)=-\infty
$$

or that $q(t)$ satisfies (2.16), hence (2.17), for some constant $C$.

The following simple consequence of Proposition 2.6 for the linear equation (1.3) does not seem to have been noted before.

COROLlaRY 2.2. Let (1.3) be nonoscillatory at $t=\infty$ and let (2.22) [or, for example, (2.6)] hold. Let $x=x(t), x^{0}(t)$ be a pair of linearly independent solutions of (1.3). Then $\int^{\infty}\left|x x^{0}\right|^{-2} d t<\infty$.

For a contrasting result, see Corollary 4.2 below.

Proof. The equation (1.2) has solutions for large $t$ and these satisfy (2.7), with $G(t, r)=r^{2}$. Thus, $r=x^{\prime} / x, r^{0}=x^{0 \prime} / x^{0}$ satisfy $\int^{\infty}\left|r-r^{0}\right|^{2} d t<\infty$. This implies the corollary, since the Wronskian of $x, x^{0}$ is a (nonzero) constant.

From Proposition 2.1, we can derive the following "comparison" theorem which, for (1.2), is equivalent to a known result [5, Exercise 7.9, p. 369] on linear second order equations (and is a generalization of theorems of Hille [7] and Wintner [10]).

Proposition 2.7. Let $q(t)$ satisfy (2.6) and let

$$
Q_{0}(t)=\int_{t}^{\infty} q_{0}(s) d s=\lim _{T \rightarrow \infty} \int_{t}^{T} q_{0}(s) d s
$$

exist. Let $G, m$ satisfy the conditions of Proposition 2.2,

$$
G(t, r) \text { is nondecreasing for } r \geqq 0 \quad \text { ( } t \text { fixed })
$$

and, if $Q(t) \geqq 0$ does not hold for large $t$, replace (2.25) by

$$
\max _{|s| \geqq r} G(t, s)=G(t, r) \text { for } t \text { fixed, } \quad r>0 .
$$


Finally, assume that (2.2) has solutions and

$$
|Q(t)| \leqq Q_{0}(t)
$$

for large t. Then (1.1) has solutions for large $t$.

The monotony conditions (2.25)-(2.26) on $G$ hold, of course, if $G(t, 0)=0, G$ is a convex function of $r$, and $G(t,-r) \leqq G(t, r)$ for $r>0$.

Proof. Let $r=r_{0}(t)$ be a solution of (2.2) for large $t$. Using the analogue of (2.8) in Proposition 2.2, let

$$
u(t)=r_{0}(t)-Q_{0}(t)=\int_{t}^{\infty} G\left(s, r_{0}(s)\right) d s \geqq 0 .
$$

Then $u^{\prime}=r_{0}^{\prime}+q_{0}(t)=-G\left(t, r_{0}\right)=-G\left(t, u+Q_{0}\right)$. Since $u, Q_{0} \geqq 0$, it follows from (2.25) that $u^{\prime} \leqq-G(t, u+|Q|)$. Thus, if we put $v=u+Q(t),(2.26)$ implies that

$$
v^{\prime}+G(t, v) \leqq-G(t, u+|Q|)+G(t, u+Q)-q \leqq-q .
$$

Hence, by Proposition 2.1, (1.1) has solutions for large $t$.

Proposition 2.8. Let $G(t, r) \geqq 0$ be a convex function of $r$ (for fixed $t)$. Let there exist constants $\gamma>0$ and $C$ such that

$$
w^{\prime}+G(t, w)+G(t,(\gamma+1) Q(t)) / \gamma=0
$$

has solutions for large $t$, where $Q(t)$ is defined by (2.13). Then (1.1) has solutions for large $t$. If $G(t, r)=|r|^{a}, a>1$, and $\gamma=1 /(a-1)$, then (2.28) becomes

$$
w^{\prime}+|w|^{a}+\left[a^{a} /(a-1)^{a-1}\right]|Q(t)|^{a}=0 .
$$

If $G(t, r)=r^{2}$, this reduces to a known result ([3], cf. [5, p. 364]). Proposition 2.8 is sharp for $G(t, r)=|r|^{a}, a>1$, in the sense that the constant $a^{a} /(a-1)^{a-1}$ cannot be replaced by a smaller constant; $c f$. the example in the next section and note that (1.4) does not have a solution for large $t$ if $q=k / t^{a /(a-1)}$ and $k>\left[1 / a^{a}(a-1)\right]^{1 /(a-1)}$.

Proof. Let $w(t)$ be a solution of $(2.28)$ for large $t$. Let $w(t)=(1+1 / \gamma) u(t)$, then (2.28) gives

$$
u^{\prime}+[\gamma G(t,(1+1 / \gamma) u)+G(t,(\gamma+1) Q(t))] /(1+\gamma)=0 .
$$

Thus, by the convexity of $G, u^{\prime}+G(t, u+Q) \leqq 0$. Finally, let $v=u+Q$, so that (2.1) holds and the conclusion follows from Proposition 2.1.

Proposition 2.9. Let $G(t, r) \geqq 0$ satisfy, for fixed $t$,

$$
G(t, r) / r \text { is nondecreasing for } 0<r<c(\leqq \infty)
$$

(e.g., let $G(t, 0)=0$ and $G(t, r)$ be convex for $0 \leqq r \leqq c$, $t$ fixed). Let $r=r^{1}(t)$ be $a$ function of class $C^{1}$ satisfying

$$
r^{1^{\circ}}+G\left(t, r^{1}\right) \leqq 0 \text { and } 0<r^{1}<c
$$


for large t. Let $q_{+}(t)=\max (q(t), 0)$ satisfy

$$
\int^{\infty} q_{+}(t) d t / r^{1}(t)<\infty
$$

Then (1.1) has solutions for large $t$.

This generalizes the fact that $\int^{\infty} t q_{+}(t) d t<\infty$ assures that (1.3) is nonoscillatory at $t=\infty$. It will be clear from the proof that $(2.31),(2.32)$ can be replaced by

$$
\begin{aligned}
& r^{1^{\prime}}+G\left(t, r^{1}\right) \leqq q_{0}(t) \text { and } 0<r^{1}<c, \\
& q_{0} \geqq 0, \quad q_{1}=q_{+}+q_{0}, \quad \text { and } \quad \int^{\infty}\left(q_{1} / r^{1}\right) d t<\infty \text {. }
\end{aligned}
$$

For the case, $G(t, r)=|r|^{a}, a>1$, see [5, p. 227]. The next section gives other refinements of Proposition 2.9.

Proof. For large $t$, define the functions

$$
u(t)=\int_{t}^{\infty}\left(q_{+} / r^{1}\right) d s, \quad v=r^{1} u, \quad V=v^{\prime}+G(t, v)+q(t) .
$$

Then $v^{\prime}=r^{1^{\prime}} u-q_{+} \leqq-G\left(t, r^{1}\right) u-q_{+}$, so that

$$
V \leqq-G\left(t, r^{1}\right) u+G\left(t, r^{1} u\right)+q-q_{+} .
$$

Note that $q-q_{+} \leqq 0$ and since $0 \leqq r^{1} u<r^{1}$ for large $t,(2.30)$ implies that $G\left(t, r^{1} u\right)$ $-G\left(t, r^{1}\right) u \leqq 0$. Hence $V \leqq 0$ for large $t$, and the desired conclusion follows from Proposition 2.1 .

3. Applications of Young's inequality. In this section, we also suppose that $t$ varies over $[0, \infty)$. Suppose that

$$
\begin{gathered}
G(t, r) \geqq 0 \text { is a convex function of } r \text { ( } t \text { fixed); } G(t, 0)=0 ; \\
G_{r}=\partial G / \partial r \text { is continuous, and increasing for } r \geqq 0 .
\end{gathered}
$$

Then, for fixed $t$, the complementary Young function $F(t, r) \geqq 0$ to $G(t, r)$ is continuous [in $(t, r)$ ], convex, and $F_{r}=\partial F / \partial r$ is continuous and increasing for $r \geqq 0$ ( $t$ fixed), $F(t, 0)=0$, and

$$
G(t, r)+F(t, s) \geqq r s \quad \text { for } r, s \geqq 0 .
$$

For fixed $t$, let $G_{-1}(t, r), F_{-1}(t, r)$ denote the inverse functions of $G, F$ for $r \geqq 0$,

$$
\begin{aligned}
& G\left(t, G_{-1}(t, r)\right)=G_{-1}(t, G(t, r))=r, \\
& F\left(t, F_{-1}(t, r)\right)=F_{-1}(t, F(t, r))=r .
\end{aligned}
$$

Other conditions which may be imposed on $G$ in this section are: the existence of a constant $c$ such that

$$
G(t,-r) / c \leqq G(t, r) \leqq c G(t,-r) \text { and } c>1
$$


or the existence of constants $K$ and $\alpha$ satisfying

$$
\left|G_{r}(t, r)\right| \leqq K|G(t, r)|^{\alpha} \text { for all } r, 0<\alpha<1 .
$$

Finally, let $G_{0}(t, s)$ be a continuous, nonnegative function for $t, s \geqq 0$ satisfying

$$
G(t, r s) \geqq G(t, r) G_{0}(t, s) \text { for } r, s \geqq 0, G_{0}(t, 0)=0 .
$$

TheOREM 3.1. Assume (3.1), (3.2), (3.5), (3.6) and (3.7). Assume that if a solution $r=r(t)$ exists for large $t$, then (2.7) holds (cf., e.g., Propositions 2.2, 2.3 or 2.6), and let $Q(t)$ be defined by Proposition 2.5 (e.g., by (2.6)). Then a necessary condition for (1.1) to have a solution for large $t$ is that

$$
\int^{\infty} t^{-2+1 / \alpha} \exp \left[-\int_{0}^{t} G_{0}\left(s, F_{-1}\left(s, Q_{+}(s)\right)\right) d s\right] d t=\infty,
$$

where $Q_{+}(t)=\max (Q(t), 0)$.

In particular, if $G(t, r)=|r|^{a}, a>1$, then all conditions on $G(t, r)$ are satisfied and (3.8) becomes

$$
\int^{\infty} t^{(2-a) /(a-1)} \exp \left\{-\left[a^{a} /(a-1)^{a-1}\right] \int_{0}^{t} Q_{+}^{a-1}(s) d s\right\} d t=\infty .
$$

Also, (3.6) is replaced by $G(t, r)=g(r)$,

$$
g^{\prime}(r)=a|r|^{a-1} \operatorname{sgn} r, \text { so that } K=a, \alpha=(a-1) / a,
$$

$$
g(r s)=s^{a} g(r), \text { so that } G_{0}(t, s)=s^{a} \text {, }
$$

and (3.3) by

$$
r s \leqq r^{a}+\left[(a-1) / a^{a /(a-1)}\right] s^{a /(a-1)} \text { for } r, s \geqq 0,
$$

so that $G_{-1}(t, r)=|r|^{1 / a}$ and

$$
F(s)=\left[(a-1) / a^{a /(a-1)}\right] s^{a /(a-1)}, \quad F_{-1}(r)=\left[a /(a-1)^{(a-1) / a}\right] r^{(a-1) / a} .
$$

If $a=2$ and $Q_{+}$is replaced by $Q$, the necessary condition (3.9) reduces to a known result ([3]; cf. [5, p. 368]), the proof of which permits the generalization of replacing $Q$ by $Q_{+}$, but not $a=2$ by $a>1$. Note that the factor $t^{(2-a) /(a-1)}$ in (3.9) is 1 if $a=2$, but is large or small for large $t$ according as $a<2$ or $a>2$. The result (3.9) is sharp in the sense that $t^{(2-a) /(a-1)}$ cannot be replaced by $t^{(2-a) /(a-1)-\varepsilon}, a^{a} /(a-1)^{a-1}$ cannot be replaced by $\left[a^{a} /(a-1)^{a-1}\right]+\varepsilon$ for any $\varepsilon>0$. In order to see this, choose $q(t)=\left[c /(a-1)-c^{a}\right] / t^{a /(a-1)}$ for $t \geqq 1$ and fixed $c$; in which case, (1.1) has the solution $r=c / t^{1 /(a-1)}$. The desired example results if $c$ maximizes $c /(a-1)-c^{a}$ for $c>0$; that is,

$$
\begin{aligned}
c=\left[1 / a^{a}(a-1)\right]^{1 /(a-1)}, \quad q(t) & =\left[1 / a^{a}(a-1)\right]^{1 /(a-1)} / t^{a /(a-1)}, \\
{\left[a^{a} /(a-1)\right] \int^{t} Q_{+}^{a-1}(s) d s } & =\text { const }+\log t^{1 /(a-1)},
\end{aligned}
$$

and the integrand in (3.9) becomes $1 / t$, up a constant factor. 
It is clear that Theorem 3.1 is contained in the following two lemmas.

LEMMA 3.1. Assume the conditions of Theorem 3.1, except (3.5) and (3.6). If (1.1) has a solution for large $t$, then

$$
\int_{t}^{\infty} G(s, r(s)) d s \leqq \text { (const) } \exp \left[-\int_{0}^{t} G_{0}\left(s, F_{-1}\left(s, Q_{+}(s)\right)\right) d s\right] .
$$

LEMmA 3.2. Assume that $G(t, r) \geqq 0$ is a convex function of $r$ (for fixed $t$ ), $G(t, 0)=0$, and that $G_{r}=\partial G / \partial r$ is continuous and satisfies (3.6). If (1.1) has solutions for large $t$, then at least one solution of (1.1) for large $t$ satisfies

$$
\int^{\infty}\left[G(t,-r(t))+t^{-1+1 / \alpha} G(t, r(t))\right] d t=\infty
$$

(in fact, at most one solution of (1.1) for large $t$ fails to satisfy (3.15)).

Proof of Lemma 3.1. Let $r=r(t)$ be a solution of (1.1) for large $t$. Then

$$
R(t)=\int_{t}^{\infty} G(s, r(s)) d s
$$

is convergent and (2.19) holds, where $Q(t)$ is defined by (2.6) or, equivalently, by (2.13), (2.19). Thus, $R^{\prime}=-G(t, r)$, where $r=R+Q$ by (2.19),

$$
R^{\prime}=-G(t, R+Q) \text {. }
$$

In order to prove (3.14), it suffices to verify

$$
R^{\prime} \leqq-R G_{0}\left(t, F_{-1}\left(t, Q_{+}\right)\right)
$$

Since $R^{\prime} \leqq 0$, this inequality is trivial at $t$-values where $Q(t) \leqq 0$ (i.e., $\left.Q_{+}(t)=0\right)$. Consider a fixed $t$-value, where $Q(t)=Q_{+}(t)>0$. Then the Young inequality (3.3) gives

$$
R+Q \geqq G_{-1}(t, R) F_{-1}(t, Q) \quad \text { or } \quad G(t, R+Q) \geqq G\left(t, G_{-1}(t, R) F_{-1}(t, Q)\right) .
$$

By (3.7), we have

$$
G(t, R+Q) \geqq G\left(t, G_{-1}(t, R)\right) G_{0}\left(t, F_{-1}(t, Q)\right)=R G_{0}\left(t, F_{-1}(t, Q)\right) .
$$

In view of (3.17), this gives (3.18) and completes the proof of Lemma 3.1.

Proof of Lemma 3.2. Let $r=r(t), r_{0}(t)$ be distinct solutions of (1.1) for large $t$, say, $t \geqq 0$. The existence and continuity of $G_{r}$ implies that solutions of (1.1) are uniquely determined by initial conditions, so that we can suppose that $r>r_{0}$. By (1.1),

$$
\left(r-r_{0}\right)^{\prime}+G(t, r)-G\left(t, r_{0}\right)=0,
$$

hence $r-r_{0}$ satisfies the linear equation

where

$$
\left(r-r_{0}\right)^{\prime}+h(t)\left(r-r_{0}\right)=0
$$

$$
h(t)=\left[G(t, r)-G\left(t, r_{0}\right)\right] /\left(r-r_{0}\right)
$$


Thus,

(3.20) $\quad r(t)-r_{0}(t)=2 c \exp \left[-\int_{0}^{t} h(s) d s\right], \quad$ where $2 c=r(0)-r_{0}(0)>0$.

By the convexity of $G, G\left(t,\left[r-r_{0}\right] / 2\right) \leqq\left[G(t, r)+G\left(t,-r_{0}\right)\right] / 2$.

Assume that (3.15) fails to hold for both $r$ and $r_{0}$. Then $\int^{\infty} G\left(t,\left[r-r_{0}\right] / 2\right) d t<\infty$ and, consequently,

$$
\int^{\infty} G\left(t, c \exp \left[-\int_{0}^{t} h(s) d s\right]\right) d t<\infty
$$

In view of (3.19), $|h(t)| \leqq \max \left|G_{r}(t, u)\right|$ for $r_{0}(t) \leqq u \leqq r(t)$. Hence, by (3.6) and the convexity of $G$,

$$
|h(t)| \leqq\left|G_{r}\left(t, r_{0}\right)\right|+\left|G_{r}(t, r)\right| \leqq K\left(\left|G\left(t, r_{0}\right)\right|^{\alpha}+|G(t, r)|^{\alpha}\right) .
$$

Since (3.15) does not hold for $r$ and $r_{0}$, let

$$
K_{1}=\int_{0}^{\infty} t^{(1-\alpha) / \alpha}\left(\left|G\left(t, r_{0}(t)\right)\right|+|G(t, r(t))|\right) d t<\infty .
$$

Then, by Hölder's inequality,

$$
\int_{1}^{t}|h(s)| d s \leqq K K_{1}^{\alpha}\left(\int_{1}^{t} s^{-1} d s\right)^{1-\alpha}=K K_{1}^{\alpha}(\log t)^{1-\alpha} .
$$

Note that (3.6) and $G(t, 0)=0$ imply that

so that

$$
|G(t, r)| \leqq[K(1-\alpha)|r|]^{1 /(1-\alpha)}
$$

$$
\int^{\infty} G\left(t, \exp \left[-c(\log t)^{1-\alpha}\right]\right) d t=\infty \quad \text { for all } c>0
$$

This contradicts (3.21) and (3.22), and proves Lemma 3.2.

The proof of Theorem 3.1 can be modified to yield still another necessary condition for the existence of solutions (1.1) for large $t$.

THEOREM 3.3. Let the conditions of Theorem 3.1 hold, except (3.7). Let $q(t) \geqq 0$. Then a necessary condition for (1.1) to have a nonnegative solution for large $t$ (cf. Proposition 2.2) is that

$$
\int^{\infty} t^{-1+1 / \alpha} G\left(t, c \exp \left[-\int_{0}^{t} F_{-1}(s, q(s)) d s\right]\right) d t=\infty
$$

for some constant $c>0$. If $G(t, r)=|r|^{a}, a>1$, (3.23) becomes

$$
\int^{\infty} t^{1 /(a-1)} \exp \left\{-\left[a^{2} /(a-1)^{1-1 / a}\right] \int^{t} q^{1-1 / a}(s) d s\right\} d t=\infty .
$$


The example following Theorem 3.1 shows that (3.24) is "sharp". Even the Riccati case, $G(t, r)=r^{2}$, of this result seems to be new and can be stated as follows:

COROllaRY 3.1. Let $q(t) \geqq 0$. Then a necessary condition for (1.3) to be nonoscillatory at $t=\infty$ is

$$
\int^{\infty} t \exp \left[-4 \int^{t} q^{1 / 2}(s) d s\right] d t=\infty
$$

Proof of Theorem 3.3. Let $r=r(t)$ be a nonnegative solution of (1.1) for $t \geqq S$ $(\geqq 0)$. We shall verify that

$$
r^{\prime} \leqq-r F_{-1}(t, q(t))
$$

which implies that

$$
0 \leqq r(t) \leqq r(S) \exp \left[-\int_{S}^{t} F_{-1}(s, q(s)) d s\right]
$$

By the Young inequality (3.3), $G(t, r)+q \geqq r F_{-1}(t, q)$. Thus (1.1) implies (3.26), hence (3.27). Thus, if (3.23) fails to hold for some $c>0$, it follows that

$$
\int^{\infty} t^{-1+1 / \alpha} G(t, r(t)) d t<\infty, \quad \text { where }-1+1 / \alpha>0 .
$$

By (3.5) and Lemma 3.2, this is impossible, and Theorem 3.3 is proved.

In view of Lemma 3.2 and the results of the next section, the following may be of interest.

Proposition 3.2. Let $G(t, r)$ be a convex function of $r$ (for fixed $t), G_{r}=\partial G / \partial r$ exists and is continuous, and (1.1) has solutions for large $t$.

( $\alpha$ ) Assume that for every $\varepsilon>0$, there exists a $c_{\varepsilon}>0$ such that

$$
\inf _{|r| \geqq \varepsilon>0}\left|G_{\tau}(t, r)\right| \geqq c_{\varepsilon}>0 .
$$

Then at most one solution $r=r(t)$ of (1.1) for large $t$ satisfies

$$
\int^{\infty}\left|G_{r}(t, r(t))\right| d t<\infty
$$

( $\beta$ ) If, in addition, $G_{r}(t, r)$ is a convex function of $r$, then at most one solution $r=r(t)$ of (1.1) for large $t$ satisfies

$$
\lim _{T \rightarrow \infty} \int^{T} G_{r}(t, r(t)) d t \text { and } \lim _{T \rightarrow \infty} \int^{T} G_{r}(t,-r(t)) d t \quad \text { exist. }
$$

Proof. On $(\alpha)$. Let $r(t)>r_{0}(t)$ be two solutions of (1.1) for large $t$, say, for $t \geqq 0$. Then (3.19)-(3.20) hold and, by the convexity of $G$,

$$
G_{r}\left(t, r_{0}(t)\right) \leqq h(t) \leqq G_{r}(t, r(t))
$$


Suppose, if possible, that (3.29) [or even the first part of (3.30)] holds for both $r(t)$ and $r_{0}(t)$. Then

Then, by (3.20),

$$
\int^{\infty}|h(t)| d t<\infty \quad\left[\text { or } \lim _{T \rightarrow \infty} \int^{T} h(t) d t \text { exists }\right] .
$$

$$
\lim _{T \rightarrow \infty}\left[r(t)-r_{0}(t)\right]=4 L>0 \text { exists. }
$$

In particular, for every large $t$, either $r(t)>L$ or $r_{0}(t)<-L$ and, by (3.28),

$$
\left|G_{r}(t, r(t))\right|+\left|G_{r}\left(t, r_{0}(t)\right)\right| \geqq c_{L}>0 .
$$

This contradicts the assumption that (3.29) holds for both $r(t)$ and $r_{0}(t)$.

On ( $\beta$ ). Suppose, if possible, that (3.30) holds for both $r(t)$ and $r_{0}(t)$. Then (3.32) holds. By the convexity of $G_{r}$,

$$
G_{r}\left(t,\left(r-r_{0}\right) / 2\right) \leqq\left[G_{r}(t, r)+G_{r}\left(t,-r_{0}\right)\right] / 2 .
$$

For large $t,\left(r-r_{0}\right) / 2>L>0$ and so, by (3.28),

$$
0<2 c_{L} \leqq G_{r}(t, r)+G_{r}\left(t,-r_{0}\right) .
$$

This contradicts the assumption that (3.30) holds for both $r$ and $r_{0}$.

4. Principal solutions and asymptotic integration. We shall assume, in this section, that $t, r$ vary over the ranges $[0, \omega),(-\infty, \infty)$, where $0<\omega \leqq \infty$, and that all functions of $t, r$, or $(t, r)$ are defined on these ranges, unless otherwise specified. The first result gives the existence of a least or principal solution of (1.1) at $t=\omega$.

Lemma 4.1. For every $T, 0<T<\omega$, let there exist a continuous, positive function $m_{T}(r)$ for large $r>0$ satisfying

$$
\begin{gathered}
m_{T}(r) \rightarrow \infty \text { as } r \rightarrow \infty \text { and } \int^{\infty} d r / m_{T}(r)<\infty, \\
G(t,-r) \geqq m_{T}(r) \text { for } 0 \leqq t \leqq T \text { and large } r>0 .
\end{gathered}
$$

Let (1.1) have solutions for t near $\omega$. Then there exists an $S_{*}, 0 \leqq S_{*}<\omega$, and a solution $r=r_{*}(t)$ of (1.1) for $S_{*}<t<\omega$ with the following properties: If $r=r(t)$ is a solution of (1.1) on $S<t<\omega$, then $S \geqq S_{*}$ and $r(t) \geqq r_{*}(t)$ for $S<t<\omega$.

The solution $r=r_{*}(t)$ of (1.1) will be called the principal solution [at $t=\omega$ ]. Any other solution $r=r(t)$ of (1.1) for $t$ near $\omega$ will be called a nonprincipal solution.

The proof of Lemma 4.1 gives a new proof for the existence of principal solutions for a linear second order equation ([8], see [5, pp. 355-357]).

Proof. Let $0 \leqq S<S+\delta<\omega$. We first show that if $c=c(S, \delta)>0$ is sufficiently large, then a solution of (1.1) with an initial value $r(S) \leqq-c$ at $t=S$ does not exist on $[S, S+\delta]$. Let $M=\max |q(t)|$ on $[S, S+\delta]$ and let $c>0$ be so large that $m_{S+o}(r)>2 M$ if $r \geqq c$. Then, for a solution of (1.i) satisfying $r(S) \leqq-c$, we have

$$
r^{\prime} \leqq-G(t, r)+M \leqq-m_{S+\delta}(-r) / 2<0
$$


on any interval of existence $[S, T] \subset[S, S+\delta]$. In which case

$$
2 \int_{c}^{\infty} d r / m_{S+\delta}(r) \geqq T-S
$$

Hence, if $c>0$ is so large that the expression on the left is less than $\delta$, then no interval $[S, T]$ of existence has a length $\delta$.

Let $r=r(t)$ be a solution of (1.1) for $t$ near $\omega$ and let $\left(S_{r}, \omega\right)$ be its maximal open interval of existence. Put

$$
S_{*}=\inf S_{r} \text { and } r_{*}(t)=\left\{\inf r(t): S_{r}>t\right\} \geqq-\infty,
$$

so that $S_{r} \geqq S_{*}$ and $r_{*}(t) \geqq r(t)$ for $t>S_{r}$ holds. The first paragraph of this proof shows that $r_{*}(t)$ is finite for $S_{*}<t<\omega$. Since the minimum of two solutions of (1.1) on a common interval of existence is again a solution of (1.1), it is easy to see that $r=r_{*}(t)$ is a solution of $(1.1)$ for $S_{*}<t<\omega$. This proves Lemma 4.1.

Lemma 4.2. Assume that

$$
G(t, r) \text { is a convex function of } r \quad \text { ( } t \text { fixed). }
$$

Let $r^{0}(t)<r^{1}(t)<r^{2}(t)$ be distinct solutions of (1.1) on a common t-interval. Then

$$
1 \leqq\left[r^{2}(t)-r^{0}(t)\right] /\left[r^{1}(t)-r^{0}(t)\right] \text { is nonincreasing; }
$$

in particular, if $r^{0}, r^{1}, r^{2}$ exist for $t$ near $\omega$, then

$$
\lim _{t \rightarrow \omega}\left[r^{2}(t)-r^{1}(t)\right] /\left[r^{1}(t)-r^{0}(t)\right] \text { exists. }
$$

It will be seen below that, under suitable conditions on $G(t, r)$ and $q(t)$, the limit (4.5) is 1 for all choices of $r^{1}(t), r^{2}(t)$ when $r^{0}(t)$ is the principal solution of (1.1); cf. Lemma 4.4 and Theorems $4.1,4.2$ below. That the limit (4.5) need not be 1 , even if the conditions of both Lemmas 4.1 and 4.2 hold and $r^{0}$ is the principal solution, follows by choosing $q(t) \equiv 0, G(t, r) \equiv g(r)$, and $g(r) \equiv r$ for $r \geqq 0$.

REMARK. Condition (4.3) and the continuity of $G$ implies that $G$ satisfies a local uniform Lipschitz condition with respect to $r$. In fact, if $0<T<\omega, R>0$, and

$$
C_{R T}=\max _{0 \leqq t \leqq T}\{|G(t, R+1)-G(t, R)|,|G(t,-R)-G(t,-R-1)|\},
$$

then, for $0 \leqq t \leqq T<\omega$ and $|r|,|s| \leqq R$,

$$
|G(t, r)-G(t, s)| \leqq C_{R T}|r-s| \text {. }
$$

Hence solutions of (1.1) are uniquely determined by initial conditions.

Proof. Let $r^{0}, r^{1}, r^{2}$ be solutions of (1.1) on some (closed) interval with left end point $T$. From (1.1),

$$
\left(r^{i}-r^{0}\right)^{\prime}=-\left[G\left(t, r^{i}\right)-G\left(t, r^{0}\right)\right] \text { for } i=1,2,
$$


and, if $c_{i}=r^{i}(T)-r^{0}(T)$,

$$
r^{i}(t)-r^{0}(t)=c_{i} \exp \int_{T}^{t}-h_{i}(s) d s,
$$

where

$$
h_{i}(t)=\left[G\left(t, r^{i}(t)\right)-G\left(t, r^{0}(t)\right)\right] /\left[r^{i}(t)-r^{0}(t)\right] .
$$

Thus

$$
\left[r^{2}(t)-r^{0}(t)\right] /\left[r^{1}(t)-r^{0}(t)\right]=\left(c_{2} / c_{1}\right) \exp \int_{T}^{t}-\left[h_{2}(s)-h_{1}(s)\right] d s .
$$

By (4.8) and $r^{0}<r^{1}<r^{2}$, it follows that $h_{2}-h_{1} \geqq 0$, and (4.4) follows.

In the remainder of this section, we shall make the following

Assumption (A). Let $G(t, r)$ be a convex function of $r$ ( $t$ fixed) and satisfy the conditions of Lemma 4.1 involving (4.1)-(4.2).

We shall use the following notation: In terms of a pair of functions $r^{0}(t)<r^{1}(t)$, put

$$
h(t, u)=\left[G\left(t, r^{0}(t)+u\right)-G\left(t, r^{0}(t)\right)\right] / u,
$$

where $h(t, 0)=G_{r}\left(t, r^{0}(t)\right)$ is the left derivate of $G$ with respect to $r$ at $r=r^{0}(t)$,

$$
H(t, w)=h(t, \Delta)-h(t, w \Delta), \quad \Delta=\Delta(t)=r^{1}(t)-r^{0}(t)>0 .
$$

Thus, we have $h(t, u)$ is a nondecreasing function of $u$,

$$
h(t, \Delta)=\left[G\left(t, r^{1}(t)\right)-G\left(t, r^{0}(t)\right)\right] /\left[r^{1}(t)-r^{0}(t)\right],
$$

$$
G_{r}\left(t, r^{0}(t)\right) \leqq h(t, u) \leqq G_{r}\left(t, r^{0}(t)+u\right) \text { for } u>0,
$$

(4.13-)

$$
G_{r}\left(t, r^{0}(t)+u\right) \leqq h(t, u) \leqq G_{r}\left(t, r^{0}(t)\right) \quad \text { for } u<0 .
$$

Proposition 4.1. The function $H$ satisfies

$$
H(t, w) \text { is nonincreasing in } w \text { and } H(t, 1)=0 \text {; }
$$

for $w>1$,

$$
(1-1 / w)\left[G_{r}\left(t, r^{1}\right)-h(t, \Delta)\right] \leqq-H(t, w) \leqq G_{r}\left(t, r^{0}+w \Delta\right)-h(t, \Delta) ;
$$

for $0<w<1$,

$$
\begin{aligned}
(1 / w-1)\left[G_{r}\left(t, r^{0}+w \Delta\right)-h(t, w \Delta)\right] & \leqq H(t, w) \leqq h(t, \Delta)-G_{r}\left(t, r^{0}\right) \\
& \leqq G_{r}\left(t, r^{1}\right)-G_{r}\left(t, r^{0}\right)
\end{aligned}
$$

and, for $w<0$,

$$
\begin{aligned}
h(t, \Delta)-G_{r}\left(t, r^{0}\right) & \leqq H(t, w) \leqq h(t, \Delta)-G_{r}\left(t, r^{0}+w \Delta\right) \\
& \leqq G_{r}\left(t, r^{1}\right)-G_{r}\left(t, r^{0}+w \Delta\right) .
\end{aligned}
$$

Proof. In view of the monotony of $h$ and $(4.13 \pm)$, only the left inequalities in (4.15), (4.16) have to be verified. Consider first (4.15), where $w>1$,

$$
\begin{aligned}
-H(t, w) & =\int_{\Delta}^{w \Delta}[d h(t, u) / d u] d u, \\
d h / d u & =u^{-2}\left\{G_{r}\left(t, r^{0}+u\right) u-\left[G\left(t, r^{0}+u\right)-G\left(t, r^{0}\right)\right]\right\} .
\end{aligned}
$$


The convexity of $G$ implies that $\{\cdots\}$ is a nondecreasing function of $u$ (e.g., if $G$ is of class $C^{2}$, the derivative of $\{\cdots\}$ with respect to $u$ is $G_{r r}\left(t, r^{0}+u\right) u \geqq 0$ for $0<\Delta \leqq u \leqq w \Delta$ ). Hence

$$
-H\left(t, w^{\prime}\right) \geqq\left\{G_{r}\left(t, r^{1}\right) \Delta-\left[G\left(t, r^{1}\right)-G\left(t, r^{0}\right)\right]\right\} \int_{\Delta}^{w \Delta} u^{-2} d u,
$$

which is the first inequality in (4.15). The first inequality in (4.16) is proved similarly.

The next lemma gives a fundamental property of the principal solution.

LEMMA 4.3. Assume (A) and that (1.1) has solutions for $t$ near $\omega$. Let $r^{0}(t)<r^{1}(t)$ be the principal and a nonprincipal solution of (1.1). Then, for fixed $w<0$,

$$
\int^{\omega} H(t, w) d t=\infty
$$

in particular,

$$
\begin{gathered}
\int^{\omega}\left\{h(t, \Delta)-G_{r}\left(t, r^{0}+w \Delta\right)\right\} d t=\infty, \\
\int^{\omega}\left\{G_{r}\left(t, r^{1}\right)-G_{r}\left(t, r^{0}+w \Delta\right)\right\} d t=\infty .
\end{gathered}
$$

Proof. Let $r=r(t)$ be an arbitrary solution of (1.1) for $t$ near $\omega$, so that $\left(r-r^{0}\right)^{\prime}+G(t, r)-G\left(t, r^{0}\right)=0$. Introduce the new dependent variable

$$
w=\left(r-r^{0}\right) /\left(r^{1}-r^{0}\right)
$$

then (1.1) becomes

$$
w^{\prime}=w H(t, w) .
$$

Suppose that, for some $w=w_{0}<0$,

$$
\int^{\omega} H\left(t, w_{0}\right) d t<\infty
$$

Then, since $0 \leqq H(t, w) \leqq H\left(t, w_{0}\right)$ for $w_{0} \leqq w \leqq 0$, it follows that for any $w_{1}, w_{0}<w_{1}$ $<0$, there exists a solution $w(t)$ of (4.21) for $t$ near satisfying $w(t) \rightarrow w_{1}$ as $t \rightarrow \omega$. (This assertion is a consequence of results of Wintner; cf. [5, pp. 273-275].) If $w_{1}<0$, so that $w(t)<0$ for large $t$, then the corresponding solution $r=r(t)$ of (1.1) satisfies $r(t)<r^{0}(t)$ for $t$ near $\omega$, by (4.20). Hence if (4.22) holds for some $w_{0}<0$, then $r=r^{0}(t)$ is not a principal solution. This contradiction proves the lemma.

Lemma 4.4. Assume (A). Let

$$
r^{\prime}+G(t, r)+q_{0}(t)=0
$$

have solutions for $t$ near $\omega, r=r^{0}(t)$ be the principal solution of (4.23), and $r=r^{1}(t)$ $\left(>r^{0}(t)\right)$ a nonprincipal solution. Let $q(t)$ satisfy

$$
\int^{\omega}\left(\left|q(t)-q_{0}(t)\right| /\left|r^{1}(t)-r^{0}(t)\right|\right) d t<\infty
$$


( $\alpha$ ) Then (1.1) has solutions $r=r(t)$ for $t$ near $\omega$ and these satisfy

$$
\begin{gathered}
\lim _{t \rightarrow \omega}\left(r-r^{0}\right) /\left(r^{1}-r^{0}\right)=L=L[r] \text { exists; } \\
L[r] \geqq 0 ;
\end{gathered}
$$

some solutions $r=r(t)$ satisfy $L[r] \geqq 1$; and the principal solution $r=r_{*}(t)$ of $(1.1)$ satisfies

$$
L[r]=0 .
$$

( $\beta$ ) If $H(t, w)$ satisfies

$$
\int^{\omega}-H(t, w) d t=\infty \text { for all fixed } w>1,
$$

then

$$
0 \leqq L[r] \leqq 1
$$

and there exist solutions $r=r(t)$ of (1.1) satisfying

$$
L[r]=1 .
$$

( $\gamma$ ) If $H(t, w)$ satisfies

$$
\int^{\omega} H(t, w) d t=\infty \quad \text { for all fixed } w, \quad 0<w<1,
$$

then

$$
\int^{\omega}\left\{G_{r}\left(t, r^{1}\right)-G_{r}\left(t, r^{0}\right)\right\} d t=\infty
$$

and every solution $r=r(t)$ of (1.1) for $t$ near $\omega$ satisfies

$$
L[r]=0 \text { or } L[r] \geqq 1 .
$$

( $\delta$ ) Assume that, for some $\varepsilon>0, w H(t, w)$ is nondecreasing in $w$ for $0 \leqq w \leqq \varepsilon$ for fixed $t$ near $\omega$ or, equivalently, that

$$
\left[G\left(t, r^{1}\right)-G\left(t, r^{0}\right)\right] /\left(r^{1}-r^{0}\right) \geqq G_{r}\left(t, r_{0}+\varepsilon \Delta\right) \text { for } t \text { near } \omega,
$$

then the principal solution $r=r_{*}(t)$ of $(1.1)$ is the only solution of $(1.1)$ for $t$ near $\omega$ satisfying $L[r]=0$.

Note that (4.24) is trivially satisfied if $q(t)=q_{0}(t)$, in which case, the lemma gives sufficient conditions, (4.28), (4.31) and (4.34), for the limit (4.5) to be 1 for all solutions $r^{0}<r^{1}<r^{2}$ of (1.1), when $r^{0}$ is the principal solution. Below we shall obtain simple conditions on $G, q_{0}$ which imply (4.28), (4.31), (4.34).

In the proof of the first part of $(\alpha)$, that concerning the existence of solutions $r=r(t)$ of (1.1) for $t$ near $\omega$ and the existence of the limit (4.25), no use is made of 
the fact that $r^{0}$ is the principal solution of (4.23). It should be remarked, however, that (4.24) may be satisfied if $r^{0}$ is the principal solution, but not otherwise.

Proof. On $(\alpha)$. Introduce the new dependent variable

$$
w=\left[r-r^{0}(t)\right] /\left[r^{1}(t)-r^{0}(t)\right],
$$

so that (1.1) becomes

$$
w^{\prime}=w H(t, w)-\left(q-q_{0}\right) /\left(r^{1}-r^{0}\right) .
$$

Consider the "Lyapunov" functions

$$
V_{ \pm}(t, w)=w \pm \int_{t}^{\omega}\left(\left|q-q_{0}\right| /\left(r^{1}-r^{0}\right)\right) d s \pm 1 / t
$$

which have the trajectory derivatives

$$
\dot{V}_{+}<0 \text { if } w>1 \text { or } w<0 \text { and } \dot{V}_{-}>0 \text { if } 0<w<1
$$

relative to (4.36). This makes it clear that (4.25) holds if, for a moment, one allows $-\infty \leqq L<\infty$. Furthermore, if a solution $w=w(t)$ of (4.36) has a value $w\left(t_{0}\right)>1$ for some large $t_{0}$, then (4.37) implies that $w(t)$ exists for $t_{0} \leqq t<\omega$ and, correspondingly, $L \geqq 1$.

For $0<\varepsilon<1$, the upper and lower boundaries of the strip $\Omega=\Omega_{T, \varepsilon}$,

$$
\Omega=\left\{(t, w): T<t<\omega, V_{+}(t,-\varepsilon)<w<V_{-}(t, \varepsilon)\right\}
$$

are strict egress points (if $T$ is sufficiently near to $\omega$ ). Hence a theorem of Ważewski [9] (cf. [5, pp. 278-280]) implies that (4.36) has a solution $w=w(t)$ for $t$ near $\omega$ such that $(t, w(t)) \in \Omega$. For this solution, $|L| \leqq \varepsilon$. Since $\varepsilon>0$ is arbitrarily small, it follows that if $r=r_{*}(t)$ is the principal solution of $(1.1)$, then $L\left[r_{*}\right] \leqq 0$. Hence (4.26) implies (4.27) for $r=r_{*}$.

We shall now verify that (4.26) holds for all solutions $r=r(t)$ of $(1.1)$ for $t$ near $\omega$. Suppose, if possible, that for some $r=r(t), L[r]<0$. Then (4.24), (4.35), and (4.18) in Lemma 4.3 imply that $L[r]=-\infty$. Since $L \geqq 1$ holds for some solutions of (1.1), it follows that the roles of $q$ and $q_{0}$ can be interchanged, that is, the analogue of (4.24),

$$
\int^{\omega}\left(\left|q(t)-q_{0}(t)\right| /\left|r_{1}(t)-r_{*}(t)\right|\right) d t<\infty
$$

holds if $r_{*}(t)$ is the principal solution and $r_{1}(t)$ is a suitably chosen nonprincipal solution of (1.1). Thus

$$
\left(r_{*}-r^{0}\right) /\left(r^{1}-r^{0}\right) \rightarrow-\infty \text { and }\left(r_{1}-r^{0}\right) /\left(r^{1}-r^{0}\right) \rightarrow L_{1}(\geqq 1) \text {, }
$$

as $t \rightarrow \infty$. By the analogue of $L\left[r_{*}\right] \leqq 0$, it follows that the limit of $\left(r^{0}-r_{*}\right) /\left(r_{1}-r_{*}\right)$, as $t \rightarrow \omega$, is nonpositive. Since $r_{*}-r^{0}<0$ for $t$ near $\omega$, this limit is 0 ,

$$
0<\left(r^{0}-r_{*}\right) /\left(r_{1}-r_{*}\right) \rightarrow 0 \text { as } t \rightarrow \omega .
$$


This and relation (4.40) give, as $t \rightarrow \omega$,

$$
0<r^{0}-r_{*}=o\left(r_{1}-r_{*}\right), \quad r_{1}=r^{0}+O\left(r^{1}-r^{0}\right)=r^{0}+o\left(r^{0}-r_{*}\right) .
$$

By the last of these relations, $r_{1}-r_{*} \sim r^{0}-r_{*}$, so that the first gives the contradiction

$$
0<r^{0}-r_{*}=o\left(r^{0}-r_{*}\right) \text { as } t \rightarrow \omega .
$$

This proves $L[r]<0$ is impossible and completes the proof of $(\alpha)$.

On ( $\beta$ ). It is clear from (4.24) and (4.37) that (4.14) and (4.28) imply that $L[r]>1$ is impossible. Thus (4.29) holds if (4.28) holds.

On $(\gamma)$. Assertion (4.33) is clear from (4.26), (4.31) and (4.37). The relation (4.32) follows from (4.16).

On ( $\delta)$. Suppose that $w H(t, w)$ is nondecreasing for $0 \leqq w \leqq \varepsilon$. Then, by (4.14), it is nondecreasing for $-\infty<w \leqq \varepsilon$. It follows that (4.36) cannot have two solutions $w=w_{1}(t), w_{2}(t)$ satisfying $w_{2}(t)-w_{1}(t)>0$ and $w_{1}, w_{2} \rightarrow 0$ as $t \rightarrow \omega$, since $0<w_{2}(t)-w_{1}(t)$ is nondecreasing.

Note that, by (4.10)-(4.11), $\partial(w H) / \partial w=h(t, \Delta)-G_{r}\left(t, r_{0}+w \Delta\right)$. Since $G_{r}$ is a nondecreasing function of $r$, it is clear that (4.34) equivalent to $\partial(w H) / \partial w \geqq 0$ for $0 \leqq w \leqq \varepsilon$ for $t$ near $\omega$. This proves $(\delta)$ and completes the proof of Lemma 4.4.

The situation is comparatively simple in the case that

$$
G(t, r) \geqq 0 \text { and } G(t, 0)=0 \text {, }
$$

$q_{0}(t)=0$, the principal solution of $(4.23)$ is $r^{0}(t) \equiv 0$, and $G(t, r)$ is independent of $t$ or, more generally, that

$$
G(t, r) \text { is nondecreasing in } t \text { (for fixed } r \text { ). }
$$

TheOREm 4.1. Let $\omega=\infty$. Assume (A), (4.41), (4.42) and that

$$
G(t, r) / r \rightarrow 0 \text { as }(t, r) \rightarrow(\infty,+0) \text {. }
$$

( $\alpha$ ) Then a solution $r=r^{1}(t) \not \equiv 0$ of

$$
r^{\prime}+G(t, r)=0
$$

for large $t$ satisfies $0<r^{1}(t) \rightarrow 0$ as $t \rightarrow \infty$ and

$$
\int^{\infty}\left\{G_{r}\left(t, r^{1}(t)\right)-G\left(t, r^{1}(t)\right) / r^{1}(t)\right\} d t=\infty,
$$

in particular,

$$
\int^{\infty} G_{r}\left(t, r^{1}(t)\right) d t=\infty
$$

also, if $0<r^{1}(t)<r^{2}(t)$ are solutions of (4.44) for large $t$, then

$$
r^{2}(t) / r^{1}(t) \rightarrow 1 \text { as } t \rightarrow \infty \text {. }
$$

Let $q(t)$ in (1.1) satisfy

$$
\int^{\infty}|q(t)| / r^{1}(t) d t<\infty
$$


( $\beta$ ) Then (1.1) has solutions $r=r(t)$ for large $t$ (by Proposition 2.9) and these satisfy

$$
\lim _{t \rightarrow \infty} r(t) / r^{1}(t)=L=L[r] \text { exists, }
$$

where (4.29) holds; (4.30) holds for some solutions and (4.27) holds for the principal solution of (1.1).

$(\gamma)$ If, in addition,

$$
G\left(t, r / w^{\prime}\right) / G(t, r) \text { is bounded as }(t, r) \rightarrow(\infty,+0)
$$

for fixed $w, 0<w<1$, then $L[r]=0$ or 1 holds for all solutions of (1.1) for large $t$.

( $\delta)$ If (4.49) is strengthened to

$$
r G_{r}(t, r) / G(t, r) \text { is bounded as }(t, r) \rightarrow(\infty,+0),
$$

then $L[r]=0$ holds only for the principal solution $r=r_{*}(t)$ of $(1.1)$.

If (1.1) is the Riccati equation (1.2), this theorem reduces to a result of Bôcher on the asymptotic integration of (1.3) under the condition $\int^{\infty} t|q| d t<\infty$; cf. [5, pp. 380-381]. Clearly, $G(t, r)=|r|^{a}, a>1$, satisfies the conditions of Theorem 4.1.

RemarK. Condition (4.49) in $(\gamma)$ is only used to assure

$$
\int^{\infty}\left\{G_{r}\left(t, r^{1}\right)-G\left(t, r^{1}\right) / r^{1}\right\}\left[G\left(t, r^{1}\right) / G\left(t, r^{1} w\right)\right] d t=\infty \text { for } 0<w<1 .
$$

Proof. On ( $\alpha)$. Note that (4.42) implies that $m_{T}(r)$ in (4.1), (4.2) can be chosen independent of $T>0$. Hence, by Proposition 2.2 with $q(t) \equiv 0$, a solution $r(t)$ of (4.44) for large $t$ satisfies $0 \leqq r^{1}(t) \rightarrow 0$, as $t \rightarrow \infty$. The inequality $r^{1}(t)>0$ follows from the local uniqueness of solutions. In order to prove (4.45), note that

$$
\int^{t} G_{r}\left(s, r^{1}(s)\right) d s \geqq-\log G\left(t, r^{1}(t)\right)+\text { const. }
$$

In fact, if $G(t, r)$ has a continuous derivative with respect to $t$, then, by (4.44),

$$
d\left[-\log G\left(t, r^{1}(t)\right)\right]=\left(-G_{t} / G+G_{r}\right) d t \leqq G_{r} d t,
$$

which gives (4.51). Also, by (4.44),

$$
\int^{t}\left[G\left(s, r^{1}(s)\right) / r^{1}(s)\right] d s=-\log r^{1}(t)+\text { const, }
$$

hence

$$
\int^{t}\left(G_{r}-G / r^{1}\right) d s \geqq-\log G\left(t, r^{1}(t)\right) / r^{1}(t)+\text { const }
$$

and the right side tends to $\infty$, by (4.43).

The existence of the limit $(\geqq 1)$ in $(4.46)$ follows from Lemma 4.2 , where $r^{0} \equiv 0$. That this limit is 1 is a consequence of the case $q=q_{0}=0$ of part $(\beta)$ in Lemma 4.4; cf. the first part of (4.15). 
On ( $\beta$ ). This follows from parts $(\alpha),(\beta)$ of Lemma 4.4. Actually, the proof of (4.26) is quite simple here for, by Proposition 2.3,

$$
r(t)=\int_{t}^{\infty} G(s, r(s)) d s+\int_{t}^{\infty} q(s) d s \geqq \int_{t}^{\infty} q(s) d s,
$$

and so, by the monotony of $r^{1}(t)>0$

$$
r(t) / r^{1}(t) \geqq-\int_{t}^{\infty}\left(|q(s)| / r^{1}(s)\right) d s \rightarrow 0, \quad t \rightarrow \infty .
$$

On $(\gamma)$. Let $0<w<1$. By the first part of (4.16) and the assumption (4.49), there is a constant $c>0$ such that for large $t$,

$$
H(t, w) \geqq c(1 / w-1)\left\{G_{r}\left(t, w r^{1}\right)-G\left(t, w r^{1}\right) / w r^{1}\right\} G\left(t, r^{1}\right) / G\left(t, w r^{1}\right)
$$

if $0<w<1$ is fixed. By analogues of (4.52) and (4.53),

Hence,

$$
\begin{aligned}
d\left[-\log G\left(t, w r^{1}(t)\right)\right] & \leqq w G_{r}\left(t, w r^{1}\right) G\left(t, r^{1}\right) / G\left(t, w r^{1}\right), \\
d\left[-\log r^{1}(t)\right] & =\left[G\left(t, w r^{1}\right) / r^{1}\right] G\left(t, r^{1}\right) / G\left(t, w r^{1}\right) .
\end{aligned}
$$

$$
\int^{t} H(s, w) d s \geqq c[(1 / w-1) w]\left\{-\log G\left(t, w r^{1}(t)\right) / r^{1}(t)+\text { const }\right\},
$$

and (4.31) follows from (4.43). Consequently, $(\gamma)$ is implied by $(\gamma)$ in Lemma 4.4.

In the corollary of the next theorem, we obtain an analogue of Theorem 4.1 for the comparison of solutions of (1.1) and (4.23), where $q_{0}(t) \not \equiv 0$. Actually, the desiderata are simple conditions on $G, q_{0}$ which imply (4.28) or (4.31), so as to make the corresponding parts of Lemma 4.4 applicable.

THEOREM 4.2. Assume (A). Let (4.23) have solutions for $t$ near $\omega$; let $r^{0}(t)$ and $r^{1}(t)$ be the principal and a nonprincipal solution of (4.23). Let $\varepsilon>0$ be small, $w_{0} \geqq 1$, and for fixed $t$ near $\omega, U=U\left(\varepsilon, t, w_{0}\right)$ be the r-interval

$$
U=U\left(\varepsilon, t, w_{0}\right)=\left\{r: r^{0}(t)-\varepsilon \Delta(t) \leqq r \leqq r^{0}(t)+w_{0} \Delta(t)\right\},
$$

where $\Delta(t)=r^{1}(t)-r^{0}(t)$. Suppose that $G_{r}(t, r)$ is convex on the interval (4.54) or, more generally, that there exists a constant $c>0$ (independent of $t$ ) such that

$$
G_{r}\left(t, r_{2}\right)-G_{r}\left(t, r_{1}\right) \geqq c\left[G_{r}\left(t, s_{2}\right)-G_{r}\left(t, s_{1}\right)\right]
$$

if $r_{1}, r_{2}, s_{1}, s_{2} \in U$ and $r_{1} \geqq s_{2}, r_{2}-r_{1} \geqq s_{2}-s_{1} \geqq 0$; e.g., that $G_{r r}=\partial^{2} G / \partial r^{2}$ exists and is continuous and

$$
G_{r r}(t, r) \geqq c G_{r r}(t, s) \quad \text { if } r>s \text { and } r, s \in U .
$$

Then (4.31) holds and, if $w_{0}>1$, then (4.28) also holds. Thus, (4.32) holds and every solution $r=r(t)$ of (4.23) for $t$ near $\omega$ satisfies (4.33) and, if $w_{0}>1$, then $L[r]$ is 0 or 1 for all such solutions. 
In view of Lemma 4.4, we obtain the following

COROllary 4.1. Let $G, q_{0}, r^{0}, r^{1}$ satisfy the conditions of Theorem 4.2 and let $q$ satisfy (4.24). Then, according as $w_{0}=1$ or $w_{0}>1$, the conclusions of parts $(\alpha),(\gamma)$ or parts $(\alpha),(\beta),(\gamma)$ of Lemma 4.4 are valid.

Since (4.56), with arbitrary $w_{0}>1$, and (4.34) are trivial for $G(t, r)=r^{2} / p(t)$, assertions $(\alpha)-(\delta)$ of Lemma 4.4 are applicable in the Riccati case

$$
r^{1}+r^{2} / p(t)+q(t)=0 .
$$

The case $G(t, r)=r^{2} / p(t)$ of Corollary 4.1 is a result of Hartman and Wintner on the asymptotic integration of

$$
\left(p(t) x^{\prime}\right)^{\prime}+q(t) x=0 ;
$$

cf. [5, p. 379]. Actually, the conclusion (4.32) in Theorem 4.2 has not been remarked earlier even in the case (4.57) and can be stated as a result on the second order linear equation (4.58).

COROLlaRY 4.2. Let $p(t)>0, q(t)$ be continuous for $0 \leqq t<\omega$ and let (4.58) be nonoscillatory at $t=\omega$. Let $x=x^{0}(t), x^{1}(t)$ be a principal, nonprincipal solution of (4.58) at $t=\omega$. Then

$$
\int^{\omega} d t / p(t)\left|x^{0}(t) x^{1}(t)\right|=\infty
$$

If $G(t, r)=|r|^{a}$ and $a \geqq 2$, then (4.56) holds with $c=1$ even if " $r, s \in U$ " is replaced by " $r>s \geqq 0$ ", so that Theorem 4.2 is applicable in the case

$$
r^{\prime}+|r|^{a}+q_{0}(t)=0, \quad q_{0}(t) \geqq 0,
$$

of (4.23) with $\omega=\infty$; cf. Proposition 2.2. The introduction of the intervals (4.54) makes Theorem 4.2 applicable in some cases where $1<a<2$. For example, consider (4.60) and

$$
q_{0}(t)=\gamma / t^{a /(a-1)}, \quad 0<\gamma<\left[1 / a^{a}(a-1)\right]^{1 /(a-1)} .
$$

Since $q_{0} \geqq 0$, Proposition 2.2 shows that the principal solution satisfies

$$
r^{0}(t) \geqq \int_{t}^{\infty} q_{0}(s) d s=\gamma /(a-1) t^{1 /(a-1)} .
$$

Also (4.60) has a pair of solutions $r=c_{0} / t^{1 /(a-1)}$ and $r=c_{1} / t^{1 /(a-1)}$, where $0<c_{0}<c_{1}$ and $c=c_{0}, c_{1}$ are the roots of $c^{a}-c /(a-1)=\gamma$. Thus $r^{1}(t)=c_{1} / t^{1 /(a-1)}$ is a nonprincipal solution. The boundedness of the ratio $r^{1} / r^{0} \geqq 1$ implies that (4.56) holds for arbitrary $w_{0}>1$, even if $1<a<2$.

Proof of Theorem 4.2. For fixed $t, u, v$ and the function (4.10), consider the difference

$$
h_{u v}(t)=h(t, v \Delta)-h(t, u \Delta)=\Delta^{-1} \int_{0}^{\Delta}\left\{G_{r}\left(t, r^{0}+s v\right)-G_{r}\left(t, r^{0}+s u\right)\right\} d s,
$$


where $\Delta=\Delta(t)=r^{1}(t)-r^{0}(t), r^{0}=r^{0}(t)$. Note that

$$
H(t, w)=h_{w 1}(t)=\Delta^{-1} \int_{0}^{\Delta}\left\{G_{r}\left(t, r^{0}+s\right)-G_{r}\left(t, r^{0}+s w\right)\right\} d s .
$$

Consider first (4.31), where $0<w<1\left(\leqq w_{0}\right)$. Choose a mesh on $[-\varepsilon, w]$,

$$
-\varepsilon=u(0)<u(1)<\cdots<u(n)=w, \text { where } u(j)-u(j-1)<1-w .
$$

Then, by $(4.55), 0 \leqq c h_{u(j-1), u(j)} \leqq H(t, w)$. Also, for $H(t,-\varepsilon)=h_{-\varepsilon, 1}(t)$,

$$
H(t,-\varepsilon)=\sum_{j=1}^{n} h_{u(j-1), u(j)}+H(t, w) \leqq(n / c+1) H(t, w),
$$

and (4.31) follows from (4.18), with $w=-\varepsilon$, in Lemma 4.3.

The case (4.28), where $1<w<w_{0}$, can be treated similarly. Choose a mesh on $[-\varepsilon, 1]$,

$$
-\varepsilon=u(0)<\cdots<u(n)=1 \text {, where } u(j)-u(j-1)<w-1 .
$$

Then, by $(4.55), 0 \leqq c h_{u(j-1), u(j)} \leqq-H(t, w)$. Thus

$$
H(t,-\varepsilon)=h_{-\varepsilon, 1}=\sum_{j=1}^{u} h_{u(j-1), u(j)} \leqq-(n / c) H(t, w),
$$

and (4.28) follows from Lemma 4.3.

The remaining conclusions of Theorem 4.2 follow from Lemma 4.4, where $q=q_{0}$.

The considerations of [2] indicate that if $\omega=\infty$, then it may be possible to replace $(4.24)$ by

$$
\sup _{0<v<\infty}(1+v)^{-1}\left|\int_{t}^{t+v}\left(\left[q(t)-q_{0}(t)\right] /\left[r^{1}(t)-r^{0}(t)\right]\right) d t\right| \rightarrow 0 \text { as } t \rightarrow \infty .
$$

This is the case under suitable conditions on $G$ and $q_{0}$.

Lemma 4.5. Assume (A) with $\omega=\infty$, let $r^{0}(t), r^{1}(t)$ be principal, nonprincipal solutions of (4.23), and let (4.61) hold. Let there exist a continuous function $m(r)$, $0 \leqq r<\infty$, such that $m(0)=0, m(r)>0$ for $r>0, m(r) \rightarrow \infty$ as $r \rightarrow \infty$, and one of the following relations hold:

$$
\begin{array}{ll}
|H(t, w) w| \geqq m(|1-w|) & \text { for } 1 / 2 \leqq w<\infty, \\
|H(t, w) w| \geqq m(|w|) & \text { for }-\infty<w \leqq 1 / 2 .
\end{array}
$$

Then (1.1) has solutions for large $t$ satisfying (4.25) and $L=1$ [or $L=0$ ] if (4.62) [or (4.63)] holds. If both (4.62), (4.63) hold, then every solution $r=r(t)$ of (1.1) for large $t$ satisfies (4.25) and $L[r]=0$ or 1 . Assertion $(\delta)$ of Lemma 4.4 is valid.

The proof will be omitted as it follows closely the proofs of (I), (II) in [2, pp. 560-566] (using the device on p. 571). It might be mentioned that (4.62), (4.63) hold when $G(t, r)=r^{2}$ and $q_{0}(t)$ is a negative constant, but not if $q_{0}(t) \equiv 0$. 


\section{REFERENCES}

1. W. J. Coles, An oscillation criterion for second-order linear differential equations, Proc. Amer. Math. Soc. 19 (1968), 755-759.

2. P. Hartman, Unrestricted solution fields of almost separable differential equations, Trans. Amer. Math. Soc. 63 (1948), 560-580.

3. - On linear second order differential equations with small coefficients, Amer. J. Math. 73 (1951), 955-962.

4. - On nonoscillatory linear differential equations of second order, Amer. J. Math. 74 (1952), 389-400.

5. - Ordinary differential equations, Wiley, New York, 1964.

6. J. W. Heidel, Global asymptotic stability of a generalized Liénard equation (to appear).

7. E. Hille, Nonoscillation theorems, Trans. Amer. Math. Soc. 64 (1948), 234-252.

8. W. Leighton and M. Morse, Singular quadratic functionals, Trans. Amer. Math. Soc. 40 (1936), 252-286.

9. T. Ważewski, Sur un principe topologique de l'examen de l'allure asymptotique des intégrales des équations differentielles ordinaires, Ann. Soc. Polon. Math. 20 (1947), 279-313.

10. A. Wintner, On the nonexistence of conjugate points, Amer. J. Math. 73 (1951), 368-380.

11. - On the comparison theorem of Kneser-Hille, Math. Scand. 5 (1957), 255-260.

THE JOHNS HOPKINS UNIVERSITY,

BALTIMORE, MARYLAND 